\title{
A study on the clinical characteristics and management of patients with mild cases of COVID- 19 in shelter hospitals from China
}

\section{Tao FENG}

Guangxi Medical University

\section{Canhua YANG}

Guangxi Medical University

\section{Guiying LI}

Guangxi Medical University

\section{Daoye LIANG}

GuangXi University of Chinese Medicine

\section{Xinju JIA}

Nanning Second Peoples Hospital

Jiping TANG

Nanning Second Peoples Hospital

\section{Xinsen ZOU}

Guangxi Medical University

Zheng Li ( $\square$ lizheng1717@126.com )

Guangxi Medical University https://orcid.org/0000-0002-6419-5228

\section{Research Article}

Keywords: Novel coronavirus pneumonia (COVID-19); mild cases ; clinical characteristics; shelter hospital; management

Posted Date: May 8th, 2020

DOl: https://doi.org/10.21203/rs.3.rs-23837/v1

License: (c) (i) This work is licensed under a Creative Commons Attribution 4.0 International License. Read Full License 


\section{Abstract}

Background: To review the clinical characteristics of patients with mild cases of novel coronavirus disease (COVID-19) pneumonia admitted to shelter hospitals and to investigate the management patterns of shelter hospitals.

Methods: We collected and analyzed the case data of 931 patients of their general conditions, main clinical presentations, outcomes, laboratory results, imaging.

Results: The average age of patients with mild cases of COVID-19 was $₫ 46.68 \pm 12.13 \rrbracket$ years. There was no significant difference in incidence between males and females. The most common clinical presentations were fever (60.79\%), cough (47.37\%), myalgia and fatigue (17.40\%), sore throat (13.86\%), sputum (13.86\%), diarrhea (9.98\%), chest distress and dyspnea (8.38\%), nasal congestion $(6.02 \%)$, and runny nose $(5.69 \%)$, while $10.10 \%$ were asymptomatic. In addition, anxiety and insomnia were present in $12.13 \%$ of cases. COVID-19 clinical type: mild, 248 cases (26.64\%); moderate, 683 cases (73.36\%). Blood biochemical examination showed that some patients exhibited WBC count $(26.32 \%)$ lower to normal. Most patients tested positive for novel coronavirus nucleic acid $(55.45 \%)$, while $44.55 \%$ tested negative. The rate of positive chest computed tomography (CT )examination findings was $94.36 \%$. The most common change were ground-glass opacities (48.70\%) and multiple patchy opacities (44.58\%). Moreover, 65 patients $(6.98 \%)$ were transferred to designated hospital, primarily due to exacerbation of novel coronavirus pneumonia (32 cases, $49.2 \%$ ).

Conclusions: COVID-19 patients in shelter hospitals were in a relatively mild overall condition and were predominantly of the moderate clinical type. Attention should be paid to asymptomatic positive patients and patients without respiratory symptoms. CT is still the main patient screening method. Shelter hospitals can carry the burden of epidemic prevention and treatment of patients with mild cases of COVID-19.

\section{Background}

In December 2019, a novel coronavirus disease (COVID-19) epidemic broke out in Wuhan and has since spread rapidly throughout China and abroad ${ }^{[1,2]}$ through person-to-person transmission ${ }^{[3]}$, a single infected individual into an otherwise susceptible population was $3.58^{[4]}$, It has been categorized as a $B$ Class infectious disease, as stipulated in "Law of the People's Republic of China on the Prevention and Treatment of Infectious Diseases", and the appropriate infectious disease prevention and control measures have been implemented. Its high incidence was due it originating in Wuhan, a densely populated area, during a time when many people travel (right before the Lunar Year). Since current medical conditions were unable to fulfill patient requirements, especially in Wuhan, Hubei, where the epidemic was most severe. However, Wuhan has successively renovated and opened 14 shelter hospitals to treat a large number of patients with mild cases of novel coronavirus pneumonia as comprehensively as possible. In this paper, 931 cases of patients with mild COVID-19 admitted to the Zhuankou shelter 
hospital in a development zone of Wuhan (Hannan District), the fixed-point hospital where the GuangxiHubei Paired Assistance Epidemic Control Medical Group was located, and their age, sex, onset characteristics, laboratory results, imaging findings, outcomes, and other indicators were analyzed, with the intention of providing a basis for diagnosis and treatment of mild cases of COVID-19. In addition, the practical work experience at the shelter hospital is reviewed, which provides reference for management of epidemic prevention at shelter hospitals.

\section{Data And Methods}

1,1 Source of cases. All observational subjects were COVID-19 patients admitted to the Zhuankou shelter hospital in a development zone of Wuhan (Hannan District) at the fixed-point hospital, where the Guangxi-Hubei Paired Assistance Epidemic Control Medical Group was located. All patients met the diagnostic criteria for clinical diagnosis of COVID-19, as specified in "Diagnosis and Treatment Protocol for Novel Coronavirus Pneumonia (5th draft edition)" issued by the National Health Commission People Republic of China ${ }^{[5]}$.

1.2 Collection of clinical data. A total of 931 patients clinically diagnosed with COVID-19 admitted to the Zhuankou shelter hospital between February 17, 2020, and March 5, 2020, were included. Relevant data, including sex, age, complete blood count, nucleic acid test results, chest CT findings and outcomes were collected from electronic medical records.

1.3 Management of shelter hospitals. Standardized management was conducted according to the guidelines in the "Shelter Hospital Operational Manual", which was organized and compiled by the Medical Administration and Management Bureau of the National Health Commission of People Republic of China.

1.4 Statistics. SPSS 20 software was used for statistical description and all data analysis. Normally distributed continuous data are shown as \pm , non-normally distributed data are shown as median (interquartile range), and categorical variables are shown as frequency (proportion). The chi-square test was used to compare proportions between the two groups. Differences with $p<0.05$ were considered statistically significant.

\section{Results}

2.1 General characteristics. The patients with a mild case of COVID-19 were, on average(46.68 \pm 12.13$)$ years old. The range was 14-75 years of age, and the patients were predominantly aged between 30-60 years old (Figure 1). The male-to-female ratio was 1:1.03 (458/473). The most common clinical presentations were fever (60.79\%), cough (47.37\%), myalgia and fatigue $(17.40 \%)$, sputum $(13.86 \%)$, chest distress and dyspnea (8.38\%), diarrhea (9.98\%), nasal congestion (6.02\%), and runny nose (5.69\%), while $10.10 \%$ were asymptomatic. In addition, anxiety and insomnia were present in $12.13 \%$ of cases. COVID-19 clinical type: mild, 248 cases (26.64\%); moderate, 683 cases (73.36\%) (Table 1). 
Table 1. Clinical symptoms of patients with mild cases of COVID-19 at Zhuankou shelter hospital $[\mathrm{n}=931$, cases $(\%)]$.

\begin{tabular}{|l|c|}
\hline Symptom & Cases \\
& \\
\hline Fever & $566 \square 60.79 \square$ \\
\hline Runny nose & $53 \square 5.69 \square$ \\
\hline Nasal congestion & $56 \square 6.02 \square$ \\
\hline Sore throat & $129 \square 13.86 \square$ \\
\hline Cough & $441 \square 47.37 \square$ \\
\hline Sputum & $129 \square 13.86 \square$ \\
\hline Chestdistress, dyspnea & $78 \square 8.38 \square$ \\
\hline Myalgia, fatigue & $162 \square 17.40 \square$ \\
\hline Vomiting & $27 \square 2.90 \square$ \\
\hline Diarrhea & $65 \square 6.98 \square$ \\
\hline Abdominal pain & $26 \square 2.80 \square$ \\
\hline Asymptomatic & $94 \square 10.10 \square$ \\
\hline Anxiety and insomnia & $113 \square 12.13 \square$ \\
\hline & \\
\hline & \\
\hline & \\
\hline & \\
\hline & \\
\hline & \\
\hline & \\
\hline & \\
\hline & \\
\hline & \\
\hline & \\
\hline & \\
\hline & \\
\hline & \\
\hline & \\
\hline & \\
\hline & \\
\hline & \\
\hline
\end{tabular}

The main symptoms were fever (60.79\%), Sore throat (13.86\%) Cough (47.37\%) Sputum (13.86\%) atypical symptoms were Myalgia, fatigue (17.40\%) Anxiety and insomnia (12.13\%) Vomiting (2.90\%) Diarrhea (6.98\%) pain (2.80\%).

2.2 Laboratory examination and chest CT. Of the 798 patients that underwent blood analysis, 210 exhibited WBC count (26.32\%) lower to normal. Of the 918 patients that completed testing for novel coronavirus nucleic acid, 509 patients tested positive (55.45\%), 409 patients tested negative (44.55\%). In addition, 13 patients' nucleic acid detection results were inconclusive. A total of 922 patients underwent chest CT examination before admission, which accounted for 411 cases $(44.58 \%)$ of multiple patchy opacities and 449 cases $(48.70 \%)$ of ground-glass opacities. 62 (6.72\%) were normal(Table 2). The characteristic CT findings of a COVID - 19 patient admitted to the square

cabin hospital are shown in (Figure2a. 2b).

Table 2. Chest CT presentation of patients with mild cases of COVID-19 at Zhuankou shelter hospital [n $=922$, cases (\%)].

\begin{tabular}{|l|l|l}
\hline CT presentation & Cases & $\begin{array}{l}\text { The main features of CT are patchy changes in interstitial } \\
\text { tissues } \llbracket 44.58 \% \square \text { Ground- }\end{array}$ \\
\hline No abnormalities & $62 \square 6.72 \square$ \\
\hline Patchy changes in interstitial tissues & $411 \square 44.58 \square$ \\
\cline { 1 - 2 } Ground-glass opacities & $449 \square 48.70 \square$ & glass opacities $\square 48.70 \% \square$.
\end{tabular}

2.3 Transfers. As of March 3, 2020, 65 patients (6.98\%) of all patients hospitalized at the shelter hospital were transferred to designated hospitals due to changes in their condition.. The main reason was exacerbation of novel coronavirus pneumonia (32 cases, $49.23 \%$ of total transfer). Other factors included: psychological factors, diabetes, heart disease, urinary system disorders, digestive system disorders, disorders of other systems and personal reasons (Table 3). Exacerbation of novel coronavirus pneumonia manifested primarily as decreased $\mathrm{SpO}_{2}$ and/or chest distress and dyspnea (19 cases), persistent or recurrent fever ( 9 cases), chest pain (4 cases), and hemoptysis ( 2 cases). 853 patients had no underlying diseases, of which 38 were transferred. 78 patients presented with underlying diseases, 27 of which were transferred. The difference between the two groups was statistically significant $(p<0.05)$. 
2.4 Shelter hospital operational aspect. The operation of the shelter hospital lasted for more 20 days from the formal treatment of patients in the shelter to the rest of the shelter. There was no cross infection, no death or relapse, and no infection among 576 medical staff and work together other shelter staff.

Table 3. Reasons for transfer of patients with mild cases of COVID-19 from Zhuankou shelter hospital [n =65, cases (\%)]

\begin{tabular}{|l|l|}
\hline Reason for transfer & Cases \\
\hline Exacerbation of COVID-19 & $32 \square 49.23 \square$ \\
\hline Psychological factors & $6 \square 9.23 \square$ \\
\hline Urinary system & $3 \square 4.62 \square$ \\
\hline Other systems & $5 \square 7.70 \square$ \\
\hline Diabetes & $6 \square 9.23 \square$ \\
\hline Digestive system & $4 \square 6.15 \square$ \\
\hline Heart disease & $6 \square 9.23 \square$ \\
\hline Personal reasons & $3 \square 4.62 \square$ \\
\hline Total & 65 \\
\hline
\end{tabular}

The deterioration of the disease became the main reason for the hospital transfer, followed by the merger of patients with underlying diseases.

\section{Discussion}

The COVID-19 pathogen is an enveloped novel coronavirus of the $\beta$ genus with polymorphic morphology and 60-140 nm diameter. It is highly homologous with the SARS virus and has been named Severe Acute Respiratory Syndrome Coronavirus 2 (SARS-CoV-2) by the International Committee on Taxonomy of Viruses ${ }^{[6]}$. COVID19 is a disease characterized primarily by lung inflammation and can also cause injury and corresponding symptoms in the intestines, liver and nervous system. Its primary pathological characteristic is bilateral diffuse alveolar injury ${ }^{[7]}$.

In the 931 cases retrospectively analyzed in this study, the age range was $14-75$ years, the patients were, predominantly, 30-60 years old and the incidence rate was similar between males and females, which is consistent with data released by the Chinese Center for Disease Control and Prevention ${ }^{[8]}$. Symptoms in patients with mild cases of COVID-19 mainly include fever, cough (dry cough), myalgia and fatigue (the four main symptoms of COVID-19) with a few upper respiratory tract symptoms, such as nasal congestion and runny nose, suggesting that the virus mainly attacks lung tissue. Studies have shown that the target of SARS-CoV-2 is ACE2, which is widely expressed in lung tissue ${ }^{[9]}$. Chest distress and dyspnea are also rare and are mainly associated with the type of disease, but 19 patients in the present study were transferred to other hospitals due to chest distress and dyspnea. This is an important reason for transfer, so this patient population must be closely monitored in clinical practice. A small number of patients also presented with diarrhea. Notably, the proportion of asymptomatic patients was $10 \%$. Attention should be given to investigating the epidemiological history of patients. Currently, known sources of infection are primarily other people infected with the novel coronavirus (including those in the incubation period or who are asymptomatic) [5], so screening and timely treatment of such patients is an essential link in epidemic prevention and control. The most common chest CT manifestations in COVID19 patients are bilateral multiple subpleural ground-glass opacities, fine reticular opacities, patchy opacities and vascular thickening ${ }^{[10,11]}$. The mild disease COVID-19 summarized in this study is consistent with this kind of imaging change, suggesting that chest CT is a screening method worthy of promotion in the population with epidemiological history, so as to reduce the missed diagnosis of asymptomatic or mild symptom patients with COVID-19. Of course, there were patients with mild cases of 
COVID-19 that suffered from anxiety and insomnia (12.13\%). The psychiatrists in our group conducted timely psychological intervention and guidance and our nursing group led a Baduanjin qigong group, and rolling television broadcasts that announced that COVID-19 was preventable and treatable and other interventions have also achieved good results.

In order to properly achieve early diagnosis and treatment of COVID-19 patients and implement collection and treatment of cases in Wuhan, Hubei Province, clinical diagnosis cases were added to the Hubei Province diagnostic criteria in the "Diagnosis and Treatment Protocol for Novel Coronavirus Pneumonia (draft 5th edition)". Namely, suspected cases with the imaging presentation of pneumonia ${ }^{[5]}$ were confirmed as clinically diagnosed cases. In the present study, the proportion of cases testing negative for novel coronavirus nucleic acid was $44.55 \%$, indicating that a very high percentage of clinically diagnosed cases were diagnosed with COVID-19. On February 12, 2020, the total number of clinically diagnosed cases in the Hubei Province increased by $13332^{[12]}$. Most of these cases were predominantly mild and were situated in Wuhan City, which greatly increased the pressure on the Wuhan medical system. In order to address this problem, the Central Steering Committee urgently mobilized 20 national medical assistance groups and medical groups from various provinces and cities, and built shelter hospitals in a short period to treat patients in a timely manner and maximize isolation and treatment of patients with mild cases.

A mobile field hospital usually uses a medical shelter as a carrier and has different medical or technical support functions. It is designed to respond to sudden disasters, such as natural disasters, shipwrecks, plane crashes, wars, terrorist incidents, public health emergencies and so on ${ }^{[13]}$. The shelter hospitals used in Wuhan for COVID-19 prevention relied on repurposed factory buildings, gymnasiums, exhibition centers, large shopping malls and other buildings with open indoor spaces as ward units. Mobile shelter hospitals are used as medical functional units and technical support units to form a modular field health facility. They are characterized by construction and transformation of a temporary treatment site at a minimal cost and in a short time in order to effectively control the source of infection and maximize patient care. Shelter hospitals in China have been utilized in emergency medical rescue operations such as the 2008 Sichuan earthquake and the 2010 Yushu earthquake in Qinghai, but this was the first time they have been used to respond to public health incidents involving infectious diseases, and there have been no other mature experiences from which to draw lessons. Therefore, in order to standardize diagnosis and treatment at shelter hospitals, the Medical Administration and Management Bureau of the National Health Commission established three shelter hospitals that were put into use at early stages and compiled a "Shelter Hospital Operational Manual", which provided an important reference for the management of subsequently established shelter hospitals. The "Shelter Hospital Operational Manual" describes in detail what a shelter hospital is, background information about its construction and general condition of shelter hospitals in Wuhan. It also lays out the criteria for patient admission, check-in and processing, pre-examination and triage, treatment protocols, transfer and processing of severely ill patients, and discharge criteria. In addition, a shelter hospital management system based on the principles of directional admission, centralized isolation, unitized partition management, standardized 
treatment and bidirectional transfer was established. Shelter hospital operating conditions were strictly implemented and the Zhuankou shelter hospital achieved "zero patient deaths and zero medical personnel infections". The extremely low transfer rate due to exacerbation of primary disease $(6.98 \%)$ also confirms the feasibility of shelter hospitals for mild cases of COVID-19 during prevention and treatment of a sudden infectious disease. However, among the transferred patients, the number of those with underlying diseases was significantly higher than of those without- the difference was statistically significant. This indicates that some patients observed in the shelter hospital were still in an exacerbated state and most have underlying diseases. Therefore, during isolation and observation, special attention should be given to patients with underlying diseases.

\section{Conclusions}

Taken together, patients with mild cases of COVID-19 have fewer symptoms and milder disease conditions and shelter hospitals can undertake their diagnosis and treatment The professional medical observation and standardized treatment they provide can reduce progression from mild to severe cases and/or critical illness of Hope and Life's shelter. At the same time, standardized management of the source of infection was performed, effectively cutting off the virus transmission route. Thus, the Wuhan shelter hospital played a vital role in the COVID-19 epidemic. Of course, unfamiliarity of medical staff in the operating room of shelter hospitals can lead to low diagnosis and treatment rates. In addition, COVID19 is highly infectious, ${ }^{[14]}$ therefore creating great psychological pressure on medical personnel, which is also a problem that requires management action.

\section{Abbreviations}

COVID-19: Coronavirus disease 2019

SARS-CoV-2: Novel severe acute respiratory syndrome coronavirus

ACE2: Angiotensin-converting enzyme 2

\section{Declarations}

\section{Ethical Approval and Consent to participate}

Not applicable.

\section{Consent for publication}

Not applicable.

\section{Availability of data and materials}

Not applicable. 


\section{Competing interests}

The authors declare that they have no competing interests.

\section{Funding}

Funding for the launch of high-level talent research in Wuming Hospital affiliated to Guangxi Medical University .

\section{Authors' contributions}

Tao Feng and Yang canhua are co-first authors. They had Data collection, proofreading, statistics and writing, Guiying Li, Daoye Liang, Xinju JIA and Jiping TANG have data collection

Corresponding authors: Xinsen Zou and Zheng Li, they had design, data analysis and paper review. All authors read and approved the final manuscript.

\section{Acknowledgements}

We are grateful to Xuan Wei, Songli Wei, and Fusun Xie, the members of the fourth Guangxi medical group, for their effort in collecting the cases in this study.

\section{Authors' information}

1.Wuming Hospital of Guangxi Medical University, Nanming, Guangxi of China

Tao Feng, Canhua Yang, Guiyin Li and Zheng Li

2.ICU, First Affiliated Hospital of Guangxi Traditional Chinese Medical University, Nanning

Daoye Liang

3.ICU, Second People's Hospital of Nanning City, Nanning,

Xinju Jia , Jiping Tang

4.Guangxi Medical University, Nanning,Guangxi of China.

Xinsen Zou

\section{References}

1. Hui DS, I Azhar E, Madani TA, et al. The continuing 2019-nCoV epidemic threat of novel coronaviruses to global health-The latest 2019 novel coronavirus outbreak in Wuhan, China [J]. Int J Infect Dis, 2020, 91:264-266. DOI: 1016/j.ijid.2020.01.009 
2. Zhu N, Zhang D, Wang W, et al. A Novel Coronavirus from Patients with Pneumonia in China, 2019 [J]. N Engl J Med, 2020,382:727-733. DOI: 1056/NEJMoa2001017

3. Chan JF, Yuan S, Kok KH, et al. A familial cluster of pneumonia associated with the 2019 novel coronavirus indicating person-to-person transmission: a study of a family cluster [J]. The Lancet, 2020, 24: S0140-6736(20)30154-9. DOI: 1016/S0140-6736(20)30154-9.

4. Chen, T., Rui, J., Wang, Q. et al.A mathematical model for simulating the phase-based transmissibility of a novel coronavirus[J]. Infect Dis Poverty 9, 24 (2020). https://org/10.1186/s40249-020-00640-3.

5. National Health Commission of the People's Republic of China. Diagnosis and treatment protocols for novel coronavirus pneumonia (draft 5th edition, revised) [EB/OL]. (2020-02-08) [Access:2020-0216]. http://www.nhc.gov.cn/xcs/zhengcwj/202002/d4b895337e19445f8d728fcaf1e3e13a.shtml

6. A.E. Gorbalenya, S.C. Baker, R.S. Baric, R.J. deGroot, C. Drosten, A.A. Gulyaeva, et al. Severe acute respiratory syndrome-related coronavirus: the species and its viruses-a statement of the Coronavirus Study Group [J]. bioRxiv (2020 Feb 11), doi https [//doi.org/10.1101/2020.02.07.937862. Google Scholar.

7. Xu Z, Shi L, Wang Y, et al. Pathological findings of COVID-19 associated with acute respiratory distress syndrome [J]. The Lancet Respiratory Medicine, 2020; 8: 420-22. doi: 10.1016/S22132600(20)30085-0. Epub 2020 Feb 25.

8. Team TNCPERE. The epidemiological characteristics of an outbreak of 2019 novel coronavirus disease [J]. China CDC Weekly, 2020, (2): 113-122.

9. Xu H, Zhong L, Deng J, et al. High expression of ACE2 receptor of 2019-nCoV on the epithelial cells of oral mucosa [J]. International Journal of Oral Science, 2020, 12(1): 8. doi: 10.1038/s41368-0200074-x.

10. Xu, X., Yu, C., Qu, J. et al. Imaging and clinical features of patients with 2019 novel coronavirus SARS-CoV-2 [J]. Eur J Nucl Med Mol Imaging (2020). 2020 May;47(5):1275-1280. doi: 10.1007/s00259-020-04735-9.

11. Bai HX, Hsieh B, Xiong Z, et al. Performance of radiologists in differentiating COVID-19 from viral pneumonia on chest CT [J]. Radiology, 2020 Mar 10:200823. doi: 10.1148/radiol.2020200823.

12. National Health Commission. Update on the novel coronavirus pneumonia epidemic as of 24:00 February 12 [EB/OL]. (2020-02-13) [Access: 2020-02-15] http://www.nhc.gov.cn/xcs/yqtb/202002/26fb16805f024382bff1de80c918368f.shtml

13. Fan B, Fan HJ, Hou SK, et al. Research progress in domestic and international mobile field hospitals [J]. Chinese Medical Equipment Journal, 2010, 31(05): 28-30. DOI:10.3969/j.issn.10038868.2010.05.010

14. Chen N, Zhou M, Dong X, et al. Epidemiological and clinical characteristics of 99 cases of 2019 Coronavirus Disease 2019 in Wuhan, China: a descriptive study [J]. The Lancet, 2020, 395(10223): 507-513. 


\section{Figures}

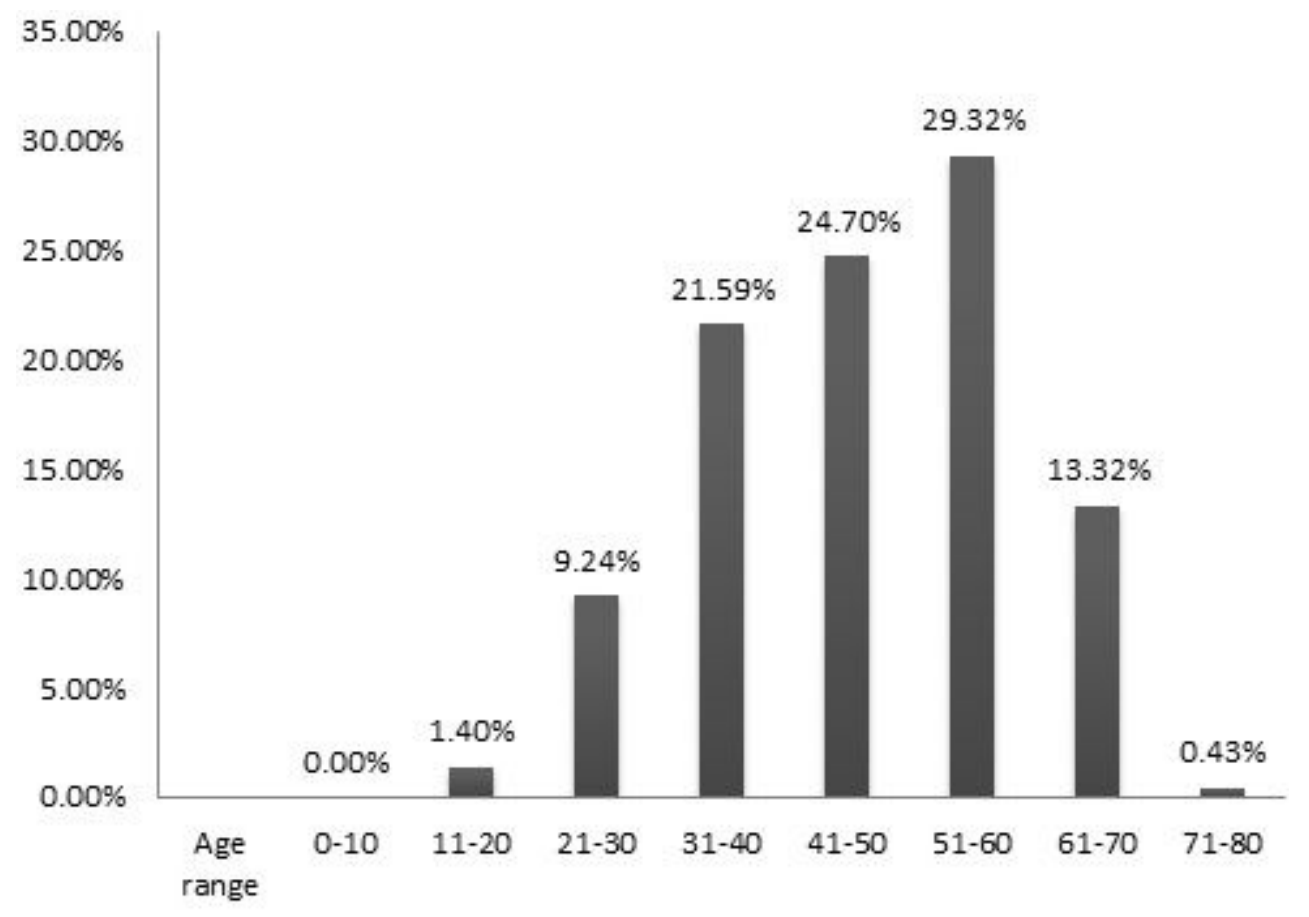

Figure 1

Age distribution of patients at onset. Patients aged 51 to 60 accounted for $29.32 \%$, patients aged 31 to 60 accounted for $75.61 \%$.

a

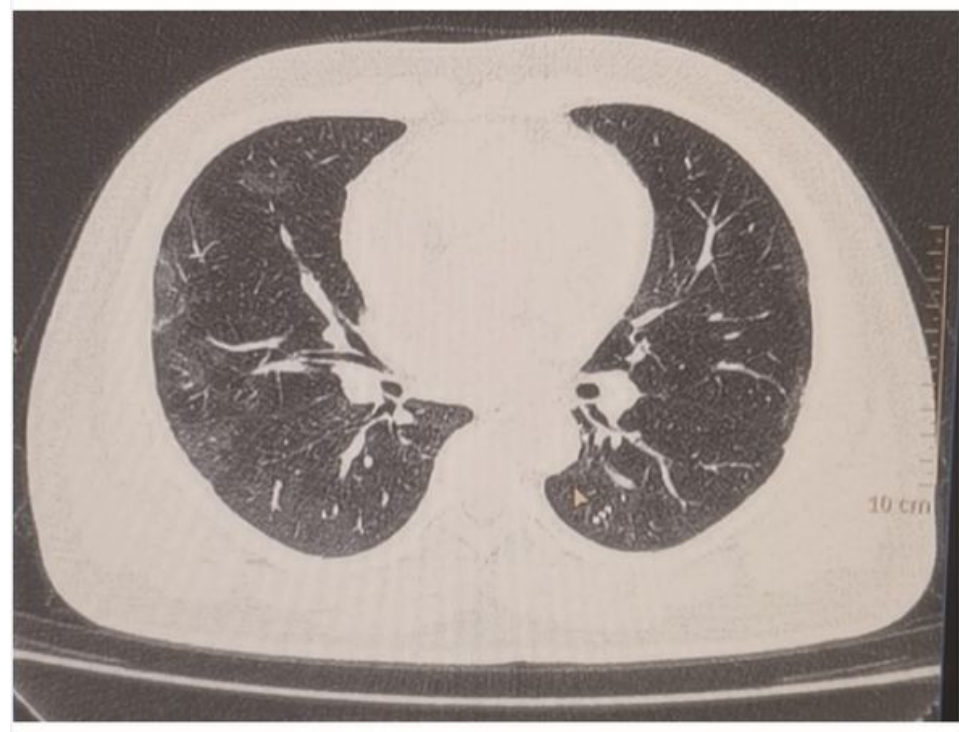

b

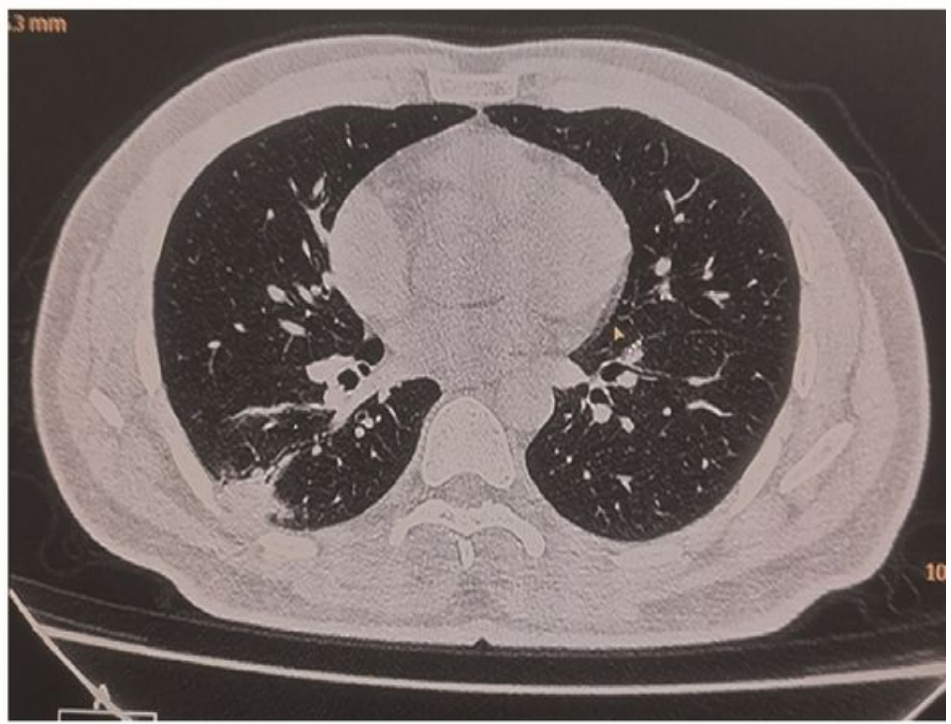

Figure 2

CT features of a COVID-19 Patient. a. COVID-19 patient 31 years, man, Fever 1 week, no shortness of breath. Ground-glass opacities on chest CT. b. Man 56- COVID-19 patient, cough for 10 days admission, no shortness of breath, Patchy changes on chest CT. 


\section{Supplementary Files}

This is a list of supplementary files associated with this preprint. Click to download.

- Slfile.xlsx 